\title{
SUBSEMIGROUPS OF FREE PRODUCTS OF SEMIGROUPS
}

\author{
by JAMES RENSHAW
}

(Received 20th July 1989)

\begin{abstract}
Given amalgams of semigroups $\left[U ; T_{i}\right]$ and $\left[U ; S_{i}\right]$ with $U \subseteq T_{i} \subseteq S_{i}$, it was proved by Howie in $[6]$ that $\prod^{*} T_{i}$ need not be embedded in $\prod_{i} S_{i}$. We use the homological techniques developed by Renshaw in [7, 9] and study three new conditions each of which imply the embeddability of the above free products.
\end{abstract}

1980 Mathematics subject classification (1985 Revision): 20M10.

Howie [6] showed that, unlike the case for groups, if $\left[U ; S_{i}\right]$ is an amalgam and if $T_{i}$ are subsemigroups of $S_{i}$ such that $\left[U ; T_{i}\right]$ is an amalgam, then $\prod_{U}^{*} T_{i}$, the free product of the amalgam $\left[U ; T_{i}\right]$, need not be embeddable in $\prod_{U}^{*} S_{i}$, the free product of the amalgam $\left[U ; S_{i}\right]$. He proved, among other things, that if $U$ and $T_{i}$ are unitary in $S_{i}$, then the free products are embeddable. We extended these results in Renshaw [8] to show that the same conclusion holds if the inclusion maps $U \rightarrow T_{i}$ and $T_{i} \rightarrow S_{i}$ are pure. We provide here three more conditions for the free products to be embeddable, one related to Hall and Howie's perfect subsemigroups, one connected with flatness of $U$-sets and one concerning right generalised inverse semigroups.

\section{The "homological" machinery}

For definitions of $U$-sets, $U$-maps etc. see for example Renshaw [7]. The category of left $U$-sets will be denoted by $U$-ENS. For convenience, we shall mainly work from now on with monoids. It is an easy matter in any case to extend these results to semigroups (see for example Renshaw [8, 9]).

Suppose that $\left[U ; S_{i}: i \in I\right]$ is an amalgam of monoids and that there exists a family of monoids $\left\{T_{i}: i \in I\right\}$ with the property that $U \subseteq T_{i} \subseteq S_{i}$ for all $i$ in $I$. We shall call the amalgam $\left[U ; T_{i}\right]$ an amalgam of submonoids of the amalgam $\left[U ; S_{i}\right]$. Our main problem is to determine some conditions under which $\prod_{U}^{*} T_{i}$, the free product of the amalgam $\left[U ; T_{i}\right]$, is embeddable in $\prod_{U}^{*} S_{i}$, the free product of the amalgam $\left[U ; S_{i}\right]$. It was shown in Renshaw [8] that we need only consider amalgams over a finite index set.

Let $X \in$ ENS- $U$. From now on, all tensor products are over $U$ unless otherwise stated. We say that $X$ is (right) flat if for all left $U$-monomorphisms $\lambda: A \rightarrow B$ the induced map $1 \otimes \lambda: X \otimes A \rightarrow X \otimes B$ is one to one. We shall say that $X$ is (right) quasi-flat if for all left $U$-monomorphisms $\lambda: A \rightarrow B$ with $B$ flat, the map $1 \otimes \lambda: X \otimes A \rightarrow X \otimes B$ is one to one 
(clearly flat implies quasi-flat). A right $U$-monomorphism $f: X \rightarrow Y$ is said to be (right) pure if for all left $U$-sets $A$, the induced map $f \otimes 1: X \otimes A \rightarrow Y \otimes A$ is one to one. A right $U$-monomorphism $f: X \rightarrow Y$ is said to be perfect if $f$ is right pure and $Y$ is right flat. (This is a generalisation of Howie's left perfect subsemigroups in Howie [5]. See also Hall [2] and Renshaw [9].) Let $f: X \rightarrow Y$ be a right $U$-map and $\lambda: A \rightarrow B$ a left $U$-map and consider the commutative diagram

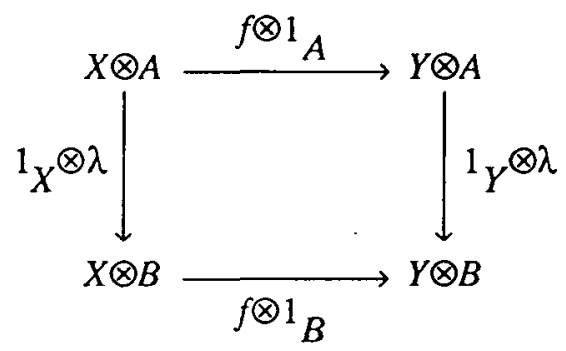

We say that the pair $(f, \lambda)$ is stable if $\operatorname{im}(f \otimes \lambda)=\operatorname{im}\left(1_{Y} \otimes \lambda\right) \cap \operatorname{im}\left(f \otimes 1_{B}\right)$. If $f: X \rightarrow Y$ is a right $U$-monomorphism and if $(f, \lambda)$ is stable for all left $U$-monomorphisms $\lambda: A \rightarrow B$ then we say that $f$ is stable. We shall say that a right $U$-monomorphism is quasi-stable if $(f, \lambda)$ is stable for all left $U$-monomorphisms $\lambda: A \rightarrow B$ with $B$ flat.

A monoid $U$ is said to be left (right) reversible if any two principal right (left) ideals of $U$ intersect. Let $X \in U$-ENS and consider the left $U$-congruence $\equiv$ on $X$ defined by $x \equiv y$ if and only if either $x=y$ or there exists $x_{1}, \ldots, x_{n} \in X, u_{1}, \ldots, u_{n}, v_{1}, \ldots, v_{n} \in U$ such that

$$
x=u_{1} x_{1}, v_{1} x_{1}=u_{2} x_{2}, v_{2} x_{2}=u_{3} x_{3}, \ldots, v_{n} x_{n}=y .
$$

There is clearly a dual definition for right $U$-sets. It is easy to demonstrate that if $x \otimes y=x^{\prime} \otimes y^{\prime}$ in $X \otimes Y$ then $x \equiv x^{\prime}$ in $X$ and $y \equiv y^{\prime}$ in $Y$. Let $f: X \rightarrow Y$ be a $U$ monomorphism and consider the right $U$-congruence on $Y$ defined by

$$
\rho_{f}=\operatorname{im} f \times \operatorname{im} f \cup 1_{Y} .
$$

We shall denote the quotient $Y / \rho_{f}$ by $Y / X$ and an element $y \rho_{f}$ by $\bar{y}$. The following results are easy to establish, see Renshaw [7].

Lemma 1.1. Let $f: X \rightarrow Y$ be a right U-monomorphism and let $A \in U-E N S$. Then

(i) $k e r(Y \otimes A \rightarrow Y / X \otimes A)=\left\{\left(f(x) \otimes a, f\left(x^{\prime}\right) \otimes a^{\prime}\right): a \equiv a^{\prime}\right\} \cup 1_{Y \otimes A}$,

(ii) if $Y / X$ is quasi-flat then $U$ is right reversible,

(iii) if $y \otimes a=f(x) \otimes a^{\prime}$ in $Y \otimes A$ then $\bar{y} \otimes a=\overline{f(x)} \otimes a$ in $Y / X \otimes A$,

(iv) if $X$ and $Y / X$ are quasi-flat then $Y$ is quasi-flat,

(v) if $Y$ is (quasi-) flat then $Y / X$ is (quasi-) flat if and only if $U$ is right reversible and $f$ is (quasi-)stable.

The following result will be required later. 
Lemma 1.2. If $f: X \rightarrow Y$ and $g: Y \rightarrow Z$ are right $U$-monomorphisms and $\lambda: A \rightarrow B$ is a left $U$-monomorphism then

(i) if $g \otimes 1: Y \otimes B \rightarrow Z \otimes B$ is one to one then $(g f, \lambda)$ is stable if both $(g, \lambda)$ and $(f, \lambda)$ are stable,

(ii) if we let $\bar{g}: Y / X \rightarrow Z / X$ be given by $\bar{g}(\bar{y})=\overline{g(y)}$, then $(\bar{g}, \lambda)$ is stable whenever $(g, \lambda)$ is stable,

(iii) if $g \otimes 1: Y \otimes B \rightarrow Z \otimes B$ is one to one and $(g f, \lambda)$ is stable then $(f, \lambda)$ is stable,

(iv) if $(g f, \lambda)$ and $(\bar{g}, \lambda)$ are stable then $(g, \lambda)$ is stable.

In particular, $g$ and $f$ are quasi-stable if and only if $g f$ and $\bar{g}$ are quasi-stable.

Proof. (i) Suppose $g f(x) \otimes b=z \otimes \lambda(a)$ in $Z \otimes B$. Then since $(g, \lambda)$ is stable, there exists $y_{1} \otimes a_{1}$ in $Y \otimes A$ such that $g f(x) \otimes b=g\left(y_{1}\right) \otimes \lambda\left(a_{1}\right)$. So $f(x) \otimes b=y_{1} \otimes \lambda\left(a_{1}\right)$ in $Y \otimes B$ and since $(f, \lambda)$ is stable, there exists $x_{2} \otimes a_{2}$ in $X \otimes A$ such that $f(x) \otimes b=$ $f\left(x_{2}\right) \otimes \lambda\left(a_{2}\right)$. Hence, $g f(x) \otimes b=g f\left(x_{2}\right) \otimes \lambda\left(a_{2}\right)$ and $(g f, \lambda)$ is stable.

(ii) Suppose that $\bar{g}(\bar{y}) \otimes b=\bar{z} \otimes \lambda(a)$ in $Z / X \otimes B$. Then from Lemma 1.1(i), we see that either

(a) $g(y) \otimes b=z \otimes \lambda(a)$ in $Z \otimes B$, from which we deduce that there exists $y_{1} \otimes a_{1}$ in $Y \otimes A$ such that $g(y) \otimes b=g\left(y_{1}\right) \otimes \lambda\left(a_{1}\right)$ and consequently $\bar{g}(\bar{y}) \otimes b=\bar{g}\left(\bar{y}_{1}\right) \otimes \lambda\left(a_{1}\right)$ and so $(\bar{g}, \lambda)$ is stable; or

(b) $g(y) \otimes b=g f\left(x_{1}\right) \otimes b_{1}, z \otimes \lambda(a)=g f\left(x_{2}\right) \otimes b_{2}$ with $b_{1} \equiv b_{2}$. By Lemma 1.1(iii) $\bar{z} \otimes$ $\lambda(a)=g f\left(x_{2}\right) \otimes \lambda(a)=\bar{g}\left(f\left(x_{2}\right)\right) \otimes \lambda(a)$ and so $(\bar{g}, \lambda)$ is stable.

(iii) If $f(x) \otimes b=y \otimes \lambda(a)$ in $Y \otimes B$ then $g(y) \otimes \lambda(a)=g f(x) \otimes b$ and so $g(y) \otimes \lambda(a)=$ $g f\left(x^{\prime}\right) \otimes \lambda\left(a^{\prime}\right)$ for some $x^{\prime}$ in $X, a^{\prime}$ in $A$, since $(g f, \lambda)$ is stable. Hence $y \otimes \lambda(a)=f\left(x^{\prime}\right) \otimes \lambda\left(a^{\prime}\right)$ as required.

(iv) Suppose $z \otimes \lambda(a)=g(y) \otimes b$ in $Z \otimes B$. Then $\bar{z} \otimes \lambda(a)=\overline{g(y)} \otimes b$ in $Z / X \otimes B$ and since $(\bar{g}, \lambda)$ is stable, we deduce that $\bar{z} \otimes \lambda(a)=\overline{g\left(y^{\prime}\right)} \otimes \lambda\left(a^{\prime}\right)$ for some $y^{\prime}$ in $Y$, $a^{\prime}$ in $A$. From Lemma 1.1(i) we see that either

(a) $z \otimes \lambda(a)=g\left(y^{\prime}\right) \otimes \lambda\left(a^{\prime}\right)$ as required, or

(b) $z \otimes \lambda(a)=g f\left(x_{1}\right) \otimes b_{1}, g\left(y^{\prime}\right) \otimes \lambda\left(a^{\prime}\right)=g f\left(x_{2}\right) \otimes b_{2}$ with $b_{1} \equiv b_{2}$. Hence, since $(g f, \lambda)$ is stable, we see that $z \otimes \lambda(a)=g f\left(x_{3}\right) \otimes \lambda\left(a_{3}\right)$ and so $(g, \lambda)$ is stable.

The following lemma generalises Lemma 1.1(i) and (iii) and the proofs are similar.

Lemma 1.3. Let $A \in E-N S-U, B \in U-E N S, X, Y \in U-E N S-U$ and $f: X \rightarrow Y a(U, U)$ monomorphism. Then

(i) $\operatorname{ker}(A \otimes Y \otimes B \rightarrow A \otimes(Y / X) \otimes B)=\left\{\left(a \otimes f(x) \otimes b, a^{\prime} \otimes f\left(x^{\prime}\right) \otimes b^{\prime}\right): a \equiv a^{\prime}, b \equiv b^{\prime}\right\} \cup 1_{A \otimes Y \otimes B}$.

(ii) if $a \otimes y \otimes b=a^{\prime} \otimes f\left(x^{\prime}\right) \otimes b^{\prime} \quad$ in $A \otimes Y \otimes B$ then $a \otimes \bar{y} \otimes b=a^{\prime} \otimes \overline{f\left(x^{\prime}\right) \otimes b}$ in $A \otimes(Y / X) \otimes B$. 
Lemma 1.4. Let $f: A \rightarrow B$ be a right $U$-monomorphism, $g: C \rightarrow D$ be $a(U, U)$ monomorphism. Then

(i) if $D, D / C$ are flat (both right and left flat) and $A$ is (quasi-)flat then $A \otimes C \rightarrow A \otimes D$ is (quasi-)stable,

(ii) if $D$ is flat and $B / A$ is (quasi-) flat than $A \otimes D \rightarrow B \otimes D$ is (quasi-)stable,

(iii) if $D, D / C$ are flat and $A, B / A$ are quasi-flat then $A \otimes C \rightarrow B \otimes D$ is a quasi-stable monomorphism and hence $(B \otimes D) /(A \otimes C)$ is quasi-flat.

Proof. (i) and (ii) are generalisations of Renshaw [7, Theorem 4.10] and the proofs are similar. In (iii), $A \otimes C \rightarrow A \otimes D$ is one to one since $A$ is quasi-flat and $A \otimes D \rightarrow B \otimes D$ is one to one since $D$ is flat. Now $A \otimes C \rightarrow A \otimes D$ is quasi-stable by (i) and $A \otimes D \rightarrow B \otimes D$ is quasi-stable by (ii) and so the composite $A \otimes C \rightarrow B \otimes D$ is quasi-stable by Lemma 1.2. Finally, $B \otimes D$ is quasi-flat since $B$ is quasi-flat (Lemma $1.1(i v)$ ) and $D$ is flat. Hence by Lemma $1.1(\mathrm{v})$ we see that $(B \otimes D) /(A \otimes C)$ is quasi-flat.

Consider the following commutative diagram of $U$-monomorphisms

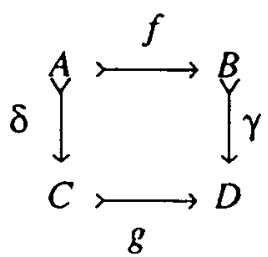

Define $\bar{\gamma}: B / A \rightarrow D / C$ by $\bar{\gamma}(\bar{b})=\overline{\gamma(b)}$. Then $\bar{\gamma}$ is well-defined and it is straightforward to show that $\bar{\gamma}$ is one to one if and only if the above diagram is a pullback.

Lemma 1.5. Suppose that the above diagram is a pullback (so that $\bar{\gamma}$ and $\bar{g}$ are one to one). Then $(D / C) /(B / A) \simeq(D / B) /(C / A)$.

Proof. Consider the following commutative diagram

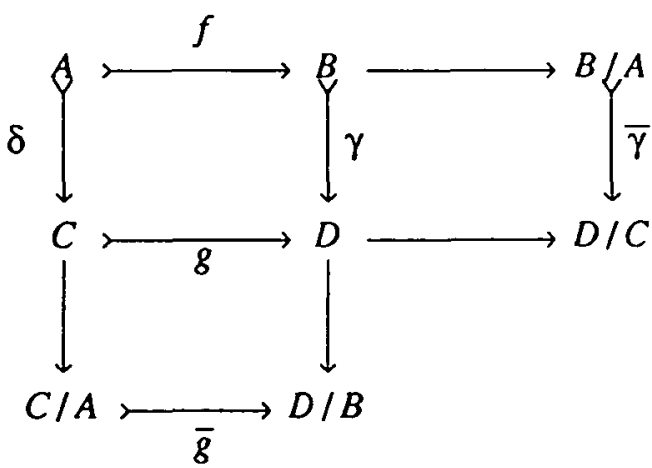


Let $\alpha: D / C \rightarrow(D / C) /(B / A)$ and $\beta: D / B \rightarrow(D / B) /(C / A)$ be the canonical maps and define $h:(D / C) /(B / A) \rightarrow(D / B) /(C / A)$ by $h(\alpha(\bar{d}))=\beta(\bar{d})$. Then $h$ is well defined, since, if $\alpha(\bar{d})=\alpha\left(d^{\prime}\right)$ then either

(i) $\bar{d}=\bar{d}^{\prime}$ in $D / C$, in which case either

(a) $d=d^{\prime}$ giving $\beta(\bar{d})=\beta\left(d^{\prime}\right)$, or

(b) $d=g(c), d^{\prime}=g\left(c^{\prime}\right)$ for some $c, c^{\prime}$ in $C$. In this case $\bar{d}=\overline{g(c)}=\bar{g}(\bar{c})$ and $\overline{d^{\prime}}=\bar{g}\left(\overline{c^{\prime}}\right)$. Hence $\beta(d)=\beta\left(\overline{d^{\prime}}\right)$; or

(ii) $\bar{d}=\bar{\gamma}(\bar{b})=\overline{\gamma(b)}$ and $\overline{d^{\prime}}=\bar{\gamma}\left(\overline{b^{\prime}}\right)=\overline{\gamma\left(b^{\prime}\right)}$ in $D / C$. Now either

(c) $d=\gamma(b)$ and so $d=\overline{\gamma(b)}=\overline{\gamma f(a)}=\overline{g \delta(a)}$ in $D / B$ (any $a \in A$ ), or

(d) $d=g(c), \gamma(b)=g\left(c^{\prime}\right)$ for some $c, c^{\prime}$ in $C$.

Either way, $\bar{d}=\bar{g}(\bar{c})$ in $D / B$ for some $c$ in $C$. Similarly, $\bar{d}^{\prime}=\bar{g}\left(\overline{c^{\prime}}\right)$ in $D / B$. Hence $\beta(\bar{d})=\beta\left(\overline{d^{\prime}}\right)$.

A similar argument shows that $h$ is one to one and it is easy to see that $h$ is onto and is a $U$-map.

Lemma 1.6. With the notation and conditions as in Lemma 1.5, suppose that $\lambda: X \rightarrow Y$ is a left $U$-monomorphism and that $(\gamma, \lambda)$ is stable and $D / C \otimes X \rightarrow D / C \otimes Y$ is one to one. Then $(\bar{g}, \lambda)$ is stable. In particular, if $D, D / B$ and $D / C$ are (quasi)-flat then so is $(D / B) /(C /$ $A)$ (and hence $(D / C) /(B / A)$ by Lemma 1.5$)$.

Proof. Suppose $\bar{g}(\bar{c}) \otimes y=\bar{d} \otimes \lambda(x)$ in $D / B \otimes Y$. Then from Lemma 1.1(i), we deduce that either

(i) $g(c) \otimes y=d \otimes \lambda(x)$ in $D \otimes Y$, or

(ii) $g(c) \otimes y=\gamma\left(b_{1}\right) \otimes y_{1}, d \otimes \lambda(x)=\gamma\left(b_{2}\right) \otimes y_{2}$ with $y_{1} \equiv y_{2}$.

In case (i), $d \otimes \lambda(x)=\overline{g(c)} \otimes \lambda(x)$ in $D / C \otimes Y$ by Lemma 1.1(iii) and so $d \otimes x=\overline{g(c)} \otimes x$ in $D / C \otimes X$. Hence, by Lemma 1.1(i) we have either

(a) $d \otimes x=g(c) \otimes x$ in $D \otimes X$, giving $d \otimes \lambda(x)=\bar{g}(\bar{c}) \otimes \lambda(x)$ as required, or

(b) $d \otimes x=g\left(c_{1}\right) \otimes x_{1}, g(c) \otimes x=g\left(c_{2}\right) \otimes x_{2}$ which again means that $d \otimes \lambda(x)=$ $\bar{g}\left(\overline{c_{1}}\right) \otimes \lambda\left(x_{1}\right)$ as required.

In case (ii), since $(\gamma, \lambda)$ is stable than $d \otimes \lambda(x)=\gamma\left(b_{3}\right) \otimes \lambda\left(x_{3}\right)$ for some $b_{3}$ in $B$ and $x_{3}$ in $X$. Hence, in $D / B \otimes Y$ we have, for any $a$ in $A, \overline{d \otimes} \lambda(x)=\gamma\left(b_{3}\right) \otimes \lambda\left(x_{3}\right)=\overline{\gamma f(a)} \otimes \lambda\left(x_{3}\right)=$ $\overline{g \delta(a)} \otimes \lambda\left(x_{3}\right)=\bar{g}(\overline{\delta(a)}) \otimes \lambda\left(x_{3}\right)$ as required.

Lemma 1.7. Let $f: X \rightarrow Y$ be a right $U$-monomorphism and $\lambda: A \rightarrow B$ a left $U$ monomorphism and suppose that all the maps in diagram (1), above, are one to one. Then diagram (1) is a pullback if and only if $(f, \lambda)$ is stable. In particular, if

(i) $f$ is perfect, or

(ii) $X$ and $Y / X$ are quasi-flat and $B$ is flat,

then diagram (1) is a pullback. 
Proof. That $(f, \lambda)$ is stable if and only if diagram (1) is a pullback follows from the note before Theorem 4.8 in Renshaw [7]. Also, if $X$ and $Y / X$ are quasi-flat then by Lemma 1.1(iv), $Y$ is quasi-flat and so all the maps in (1) are one to one. Hence by Lemma 1.1(v) diagram (1) is a pullback. If $f$ is (right) perfect then again all the maps in (1) are one to one. To show that $(f, \lambda)$ is stable, we generalise (Renshaw [9, Lemma 4.15]). Consider the following pushout diagram (in $U$-ENS):

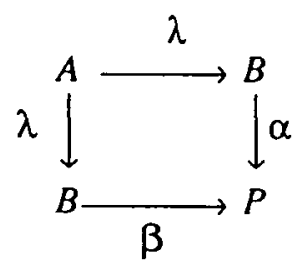

Since tensor products preserve pushouts, then

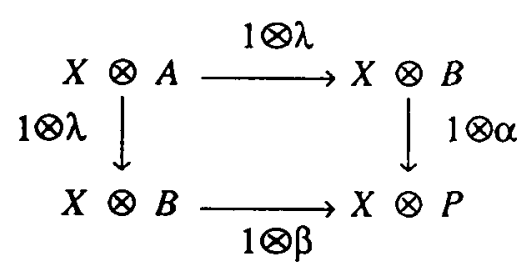

is also a pushout (and hence, since $1 \otimes \lambda$ is one to one, also a pullback). It is a pushout in ENS. Now if $y \otimes \lambda(a)=f(x) \otimes b$ in $Y \otimes B$ then

$$
f(x) \otimes \alpha(b)=y \otimes \alpha \lambda(a)=y \otimes \beta \lambda(a)=f(x) \otimes \beta(b) \text { in } Y \otimes P .
$$

Since $f$ is (right) pure then $x \otimes \alpha(b)=x \otimes \beta(b)$ in $X \otimes P$ and so (since pushouts of monomorphisms along monmorphisms are also pullbacks in ENS) there exists $x^{\prime} \otimes a^{\prime}$ in $X \otimes A$ with $x \otimes b=x^{\prime} \otimes \lambda\left(a^{\prime}\right)$ in $X \otimes B$ which means $y \otimes \lambda(a)=f\left(x^{\prime}\right) \otimes \lambda\left(a^{\prime}\right)$ in $Y \otimes B$ as required.

The following rather technical result, which was proved in Renshaw [8, Lemmas 2.2 and 2.3], forms the backbone of the machinery we need.

Lemma 1.8. Consider the following commutative diagrams
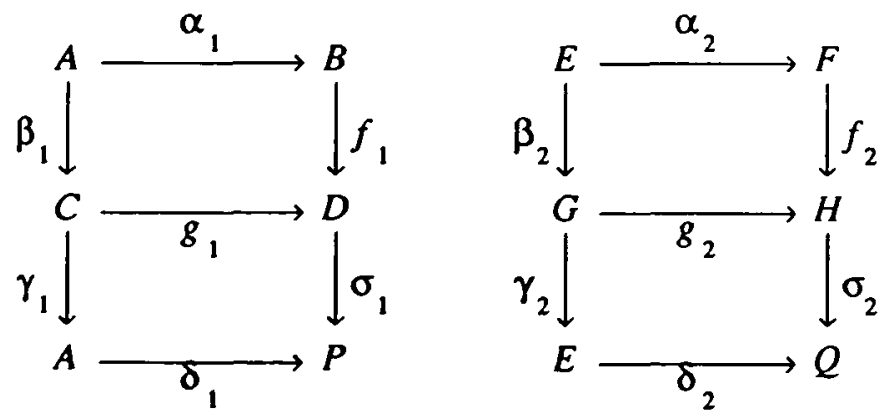
where the top squares are pullbacks and the bottom squares are pushouts and where $\gamma_{1} \beta_{1}=1_{A}, \gamma_{2} \beta_{2}=1_{E}$ and $f_{1}, g_{1}, f_{2}$ and $g_{s}$ are all one to one. Suppose we have "connecting" monomorphisms $\varphi: A \rightarrow E, \vartheta: C \rightarrow G, \varepsilon: B \rightarrow F$ and $\psi: D \rightarrow H$ such that the completed diagram

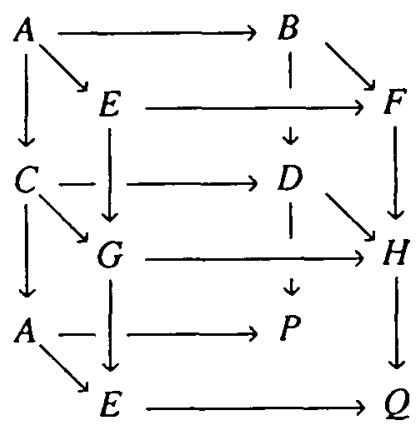

commutes and such that
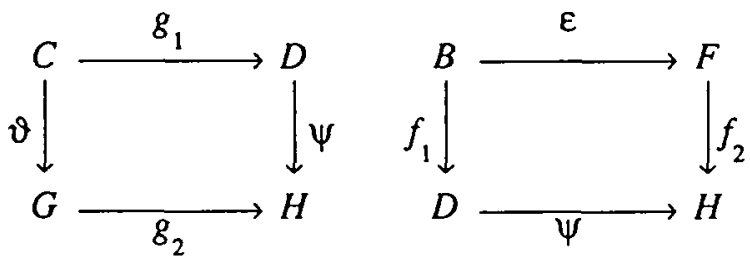

are pullbacks. Then there exists a unique monomorphism $h: P \rightarrow Q$ such that the bottom "cube" in (*) commutes and such that

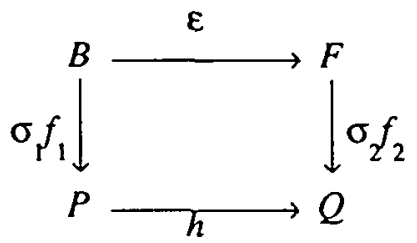

is also a pullback.

Recall that if $U$ is a submonoid of a monoid $S$ and if $X \in$ ENS-S, $Y \in$ ENS- $U$ and $f: X \rightarrow Y$ is a $U$-map then the free $S$-extension of $X$ and $Y$ consists of a right $S$-set, $F(S ; X, Y)$, together with a right $U$-map $g: Y \rightarrow F(S ; X, Y)$ such that the composite $g \circ f: X \rightarrow F(S ; X, Y)$ is a right $S$-map and such that the pair $(F(S ; X, Y), g)$ is universal with respect to this property. It was shown in Renshaw [9, Theorem 4.19] that $F(S ; X, Y)$ is the pushout in the category ENS-S of the diagram

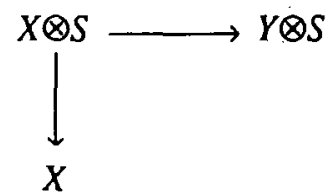


The idea behind the next two theorems is to take a diagram of the form

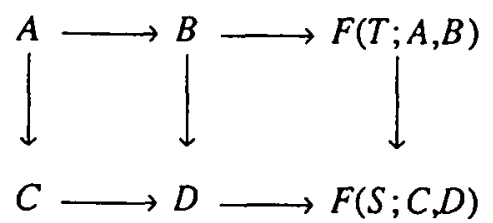

and show that for some monoids $U \subseteq T \subseteq S$, if the first square has a particular property, $P$, say, then so does the second square. This will form the basis for an inductive process in Section 2.

Theorem 1.9. Let $U \subseteq T \subseteq S$ with $U \rightarrow T$ and $U \rightarrow S$ perfect as right $U$-monomorphisms. Then $T \rightarrow S$ is perfect if and only if the following condition holds:

Whenever $A \in E N S-T, B, D \in E N S-U$ and $C \in E N S-S$ and whenever $\alpha_{1}: A \rightarrow B, \alpha_{2}: C \rightarrow D$, $\delta: A \rightarrow C$ and $\varepsilon: B \rightarrow D$ are perfect $U$-monomorphisms such that for all left $U$-sets $X$ the commutative diagram

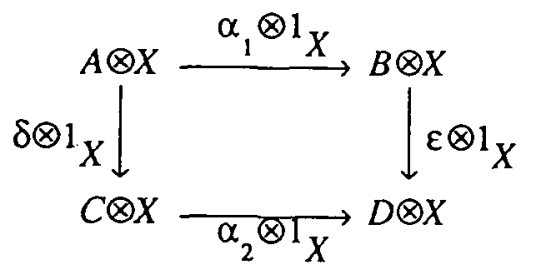

is a pullback, then the canonical maps $\beta_{1}: B \rightarrow F(T ; A, B)$ and $\beta_{2}: D \rightarrow F(S ; C, D)$ are perfect $U$-monomorphisms and there exists a unique perfect $U$-monomorphism $\psi: F(T ; A, B) \rightarrow F(S ; C, D)$ such that the commutative diagram

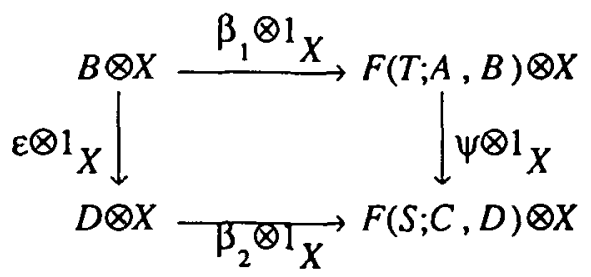

is also a pullback for all left $U$-sets $X$.

(In other words the property $P$ mentioned above is: "all maps in the square are perfect and 'tensoring' on the right by any $X$ gives a pullback".)

Proof. For the sake of brevity let $P=(T ; A, B)$ and $Q=F(S ; C, D)$. Suppose that the inclusion $T \rightarrow S$ is perfect. From Renshaw [10, Theorem V.1.3] (the proof is similar to Theorem 4.20 in Renshaw [9]) we can deduce immediately that the canonical maps $B \rightarrow P$ and $D \rightarrow Q$ are perfect monomorphisms. In particular, $P$ and $Q$ are right flat. We apply Lemma 1.8 to the diagram: 


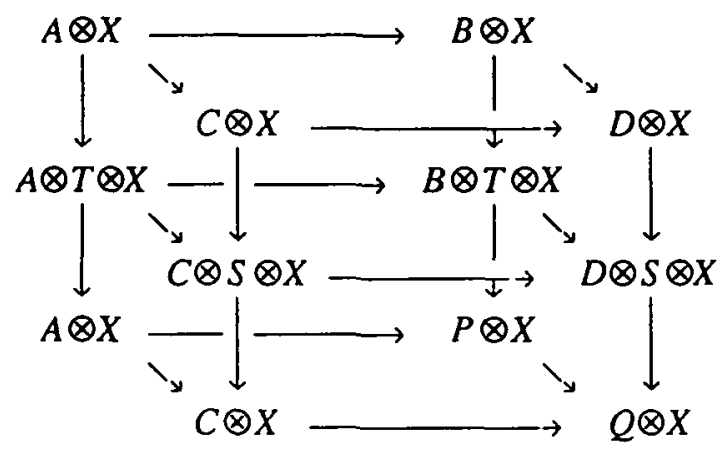

This is reasonably straightforward. The only part that requires special mention is that the commutative diagrams
(a) $A \otimes X$ $B \otimes X$<smiles>C1CCC1</smiles>

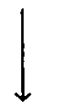
$A \otimes T \otimes X$ $B \otimes T \otimes X$

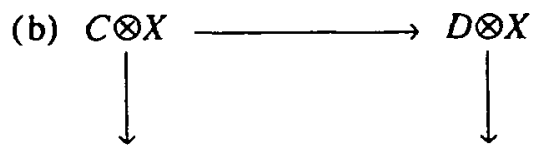
(c) $A$

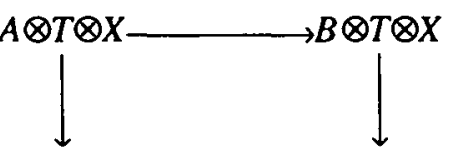
$C \otimes S \otimes X \longrightarrow D \otimes S \otimes X$

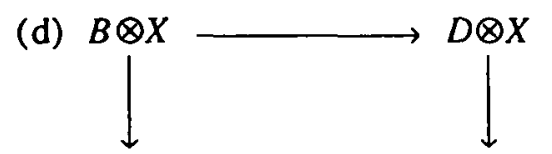
$B \otimes T \otimes X$ $D \otimes S \otimes X$

are all pullbacks. In fact diagrams (a) and (b) are pullbacks by Lemma 1.7; we can "redraw" diagram (c)

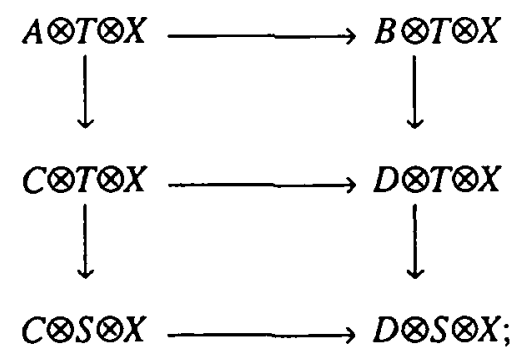

the top square is a pullback, by assumption and the bottom square is a pullback by Lemma 1.7; diagram (d) can be "redrawn"

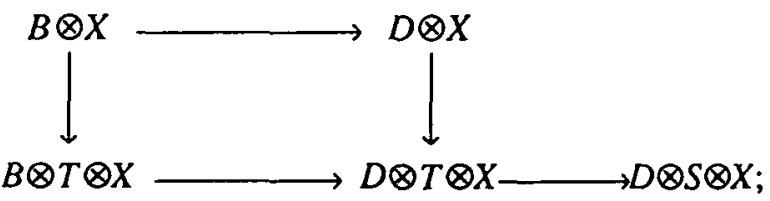


the square is a pullback by Lemma 1.7 and the map $D \otimes T \otimes X \rightarrow D \otimes S \otimes X$ is one to one since $T \rightarrow S$ is right pure and $D$ is right flat. Hence, by Lemma 1.8 , we deduce that $P \otimes X \rightarrow Q \otimes X$ is one to one and so $P \rightarrow Q$ is perfect and the commutative diagram

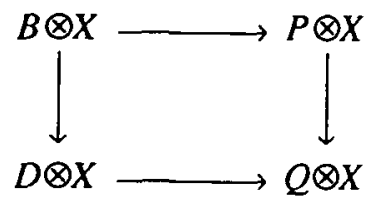

is a pullback.

Conversely, let $A=T, B=T \cup U, C=T \otimes S$ and $D=T \otimes S \cup U$ in the statement of the theorem. Then it is a straightforward matter to check that the given conditions hold. Now it is easy to establish that $F(T ; A, B)=T \cup T$ and $F(S ; C, D)=T \otimes S \cup S$ and so we can deduce that $T \cup T \rightarrow T \otimes S \cup S$ is perfect and so in particular the inclusion $T \rightarrow S$ is perfect.

The next result is of a similar nature.

Theorem 1.10. Let $U \subseteq T \subseteq S$ and suppose that $S, T, S / U, T / U$ and $S / T$ are all flat. Whenever $A \in E N S-T, B, D \in E N S-U$ and $C \in E N S-S$ and whenever $\alpha_{1}: A \rightarrow B, \alpha_{2}: C \rightarrow D$, $\delta: A \rightarrow C$ and $\varepsilon: B \rightarrow D$ are $U$-monomorphisms such that $A, B / A, C, D / C, C / A$ and $D / B$ are all quasi-flat and for all flat left $U$-sets $X$, the commutative diagram

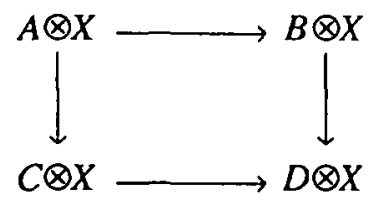

is a pullback, then there exists a unique $U$-monomorphism $\psi: F(T ; A, B) \rightarrow F(S ; C, D)$ such that $B, F(T ; A, B) / B, D, F(S ; C, D) / D$ and $F(S ; C, D) / F(T ; A, B)$ are all quasi-flat and the commutative diagram

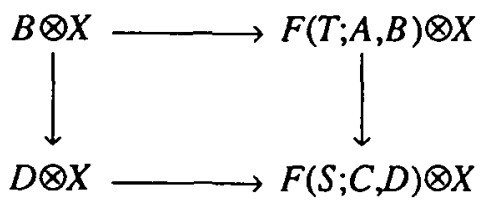

is also a pullback for all flat left $U$-sets $X$.

Proof. Again we let $P=F(T ; A, B)$ and $Q=F(S ; C, D)$. It follows immediately from Renshaw [7, Theorem 4.15] that the canonical maps $B \rightarrow P$ and $D \rightarrow Q$ are one to one and that $B, P / B, D, Q / D$ (and hence by Lemma 1.1(iv) $\mathrm{P}$ and $Q$ ) are all quasi-flat. If we apply Lemma 1.8 to diagram (2) as before we can deduce that there is a unique monomorphism $P \rightarrow Q$ such that (4) is a pullback. Finally, we need to show that $Q / P$ is quasi-flat. To this end, notice that $(D / B)$ is quasi-flat (by assumption) and so from 
Lemma 1.1 (iv) $Q / P$ will be quasi-flat if we can show that $(Q / P) /(D / B)$ is quasi-flat. Now from Lemma $1.5(Q / P) /(D / B) \simeq(Q / D) /(P / B)$. Also, from Renshaw [7, Lemma 3.10] (see also Renshaw [7, the proof of Theorem 4.15]), $Q / D \simeq D \otimes S / L$ and $P / B \simeq B \otimes T / K$ where $L$ and $K$ are, respectively, the pushouts of the following diagrams
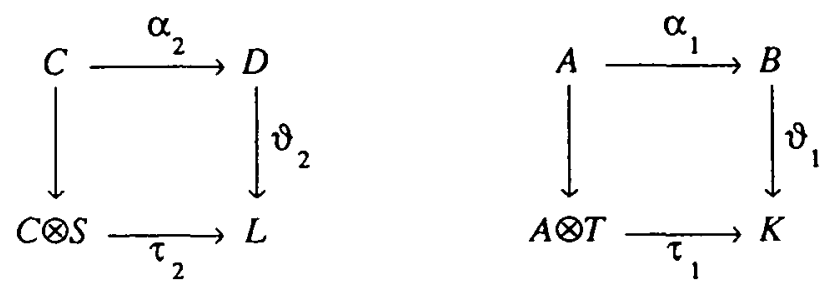

With Lemma 1.6 in mind, consider the commutative diagram

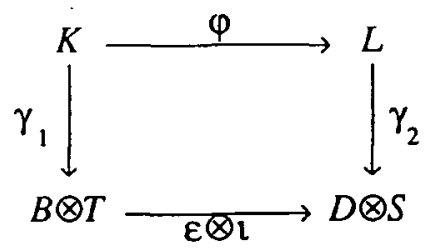

It is straightforward to show that $\gamma_{1}, \gamma_{2}$ and $\varphi$ are defined by

$$
\begin{gathered}
\gamma_{1}(k)=\left\{\begin{array}{lll}
b \otimes 1 & ; & k=\vartheta_{1}(b) \\
\alpha_{1}(a) \otimes t ; & k & =\tau_{1}(a \otimes t)
\end{array} \quad \gamma_{2}(l)= \begin{cases}d \otimes 1 ; & \quad l=\vartheta_{2}(d) \\
\alpha_{2}(c) \otimes s ; & l=\tau_{2}(c \otimes s)\end{cases} \right. \\
\varphi(k)= \begin{cases}\vartheta_{2}(\varepsilon(b)) ; & k=\vartheta_{1}(b) \\
\tau_{2}(\delta(a) \otimes t) ; & k=\tau_{1}(a \otimes t)\end{cases}
\end{gathered}
$$

We need to show that this diagram is a pullback, that all the maps in the diagram are one to one and that $D \otimes S,(D \otimes S) / L$ and $(D \otimes S) /(B \otimes T)$ are all quasi-flat. That $\gamma_{1}$ and $\gamma_{2}$ are one to one follows from Renshaw [7, Lemma 3.9] and the fact that $\left(\alpha_{1}, U \rightarrow T\right)$ and $\left(\alpha_{2}, U \rightarrow S\right)$ are stable and $\varepsilon \otimes l$ is one to one since it is the composite of the one to one maps $B \otimes T \rightarrow D \otimes T$ and $D \otimes T \rightarrow D \otimes S$. To show that $\varphi$ is one to one, suppose that $\varphi(k)=\varphi\left(k^{\prime}\right)$. There are essentially three cases to consider

(a) $k=\vartheta_{1}(b)$ and $k^{\prime}=\vartheta_{1}\left(b^{\prime}\right)$;

(b) $k=\tau_{1}(a \otimes t)$ and $k^{\prime}=\tau_{1}\left(a^{\prime} \otimes t^{\prime}\right)$; and

(c) $k=\vartheta_{1}(b)$ and $k^{\prime}=\tau_{1}\left(a^{\prime} \otimes t^{\prime}\right)$.

In cases (a) and (b), we can easily deduce that $k=k^{\prime}$ when we note that $\tau_{2}, \vartheta_{2}, \varepsilon$ and $\delta \otimes 1$ are all one to one. In case (c) we have $\vartheta_{2} \varepsilon(b)=\tau_{2}\left(\delta\left(a^{\prime}\right) \otimes t^{\prime}\right)$ and so, since the diagrams in (5) are also pullback diagrams, there exists $c$ in $C$ with $\varepsilon(b)=\alpha_{2}(c)$ in $D$ and $\delta\left(a^{\prime}\right) \otimes t^{\prime}=c \otimes 1$ in $C \otimes S$ (and so in $C \otimes T$ ). Since (3) is a pullback when $X=U$, we see 
that $b=\alpha_{1}\left(a_{1}\right)$ and $c=\delta\left(a_{1}\right)$ for some $a_{1}$ in $A$. Hence $a^{\prime} \otimes t^{\prime}=a_{1} \otimes 1$ in $A \otimes T$ and so $k=\vartheta_{1}(b)=\vartheta_{1} \alpha_{1}\left(a_{1}\right)=\tau_{1}\left(a_{1} \otimes 1\right)=\tau_{1}\left(a^{\prime} \otimes t^{\prime}\right)=k^{\prime}$ as required. Now $D \otimes S$ is quasi-flat since $D$ is quasi-flat and $S$ is flat, $(D \otimes S) / L$ is quasi-flat since it is isomorphic to $Q / D$ and from Lemma $1.4($ iii) we see that $(D \otimes S) /(B \otimes T)$ is quasi-flat since $S$ and $S / T$ are flat and $B$ and $D / B$ are quasi-flat. Finally, we show that the above diagram is a pullback. Suppose then that $\gamma_{2}(l)=\varepsilon(b) \otimes t$ in $D \otimes S$. Then either

(a) $d \otimes 1=\varepsilon(b) \otimes t$ if $l=\vartheta_{2}(d)$, or

(b) $\alpha_{2}(c) \otimes s=\varepsilon(b) \otimes t$ if $l=\tau_{2}(c \otimes s)$.

In case $(\mathrm{a}), d \otimes 1=\varepsilon(b) \otimes t$ in $D \otimes T$ since $D$ is quasi-flat and so since $\varepsilon$ is quasi-stable (and so $(\varepsilon, U \rightarrow T)$ is stable) then $\varepsilon(b) \otimes t=\varepsilon\left(b^{\prime}\right) \otimes 1$ in $D \otimes T$ for some $b^{\prime}$ in $B$. Hence $b \otimes t=b^{\prime} \otimes 1=\gamma_{1}\left(\vartheta_{1}\left(b^{\prime}\right)\right)$ and $l=\vartheta_{2}(d)=\vartheta_{2}\left(\varepsilon\left(b^{\prime}\right)\right)=\varphi\left(\vartheta_{1}\left(b^{\prime}\right)\right)$ as required.

In case (b), since $\alpha_{2}$ is quasi-stable, then $\varepsilon(b) \otimes t=\alpha_{2}\left(c_{1}\right) \otimes t_{1}$ in $D \otimes S$ (and so in $D \otimes T)$. Notice also that $c \otimes s=c_{1} \otimes t_{1}$ in $C \otimes S$. Now, putting $X=T$ in diagram (3) we deduce that $b \otimes t=\alpha_{1}\left(a_{2}\right) \otimes t_{2}=\gamma_{1}\left(\tau_{1}\left(a_{2} \otimes t_{2}\right)\right)$ and $c_{1} \otimes t_{1}=\delta\left(a_{2}\right) \otimes t_{2}$ for some $a_{2}$ in $A$, $t_{2}$ in $T$. Consequently $\varphi\left(\tau_{1}\left(a_{2} \otimes t_{2}\right)\right)=\tau_{2}\left(\delta\left(a_{2}\right) \otimes t_{2}\right)=\tau_{2}\left(c_{1} \otimes t_{1}\right)=\tau_{2}(c \otimes s)=l$.

Hence, by Lemma $1.6,(D \otimes S / L) /(B \otimes T / K) \simeq(Q / D) /(P / B) \simeq(Q / P) /(D / B)$ is quasi-flat and the theorem is proved.

\section{Subsemigroups of free products}

Let $\left[U ; S_{1}, S_{2}\right]$ be an amalgam of monoids. Define $W_{1}=S_{1}, W_{2}=S_{1} \otimes S_{2}$ and $f_{1}: W_{1} \rightarrow W_{2}$ by $f_{1}\left(s_{1}\right)=s_{1} \otimes 1$. Now define, inductively, $W_{n}=F\left(S_{i} ; W_{n-2}, W_{n-1}\right)$ $(i \equiv n(\bmod 2))$ and let $f_{n-1}: W_{n-1} \rightarrow W_{n}$ be the canonical map. It was shown in Renshaw [9] that there exist maps $\varphi_{n}: W_{n} \rightarrow S_{1} *{ }_{U} S_{2}$ for $n=1,2, \ldots$ such that $\varphi_{n} \circ f_{n-1}=\varphi_{n-1}(n \geqq 2)$ and such that the system $\left(S_{1}{ }_{U} S_{2}, \varphi_{n}\right)$ is the direct limit in the category of $U$-sets of $\left(W_{n}, f_{n}\right)$. It is clear that if $\left[U ; T_{1}, T_{2}\right]$ is an amalgam of submonoids then a similar construction, say $\left(Z_{n}, g_{n}\right)$, can be made. So we can construct a commutative diagram

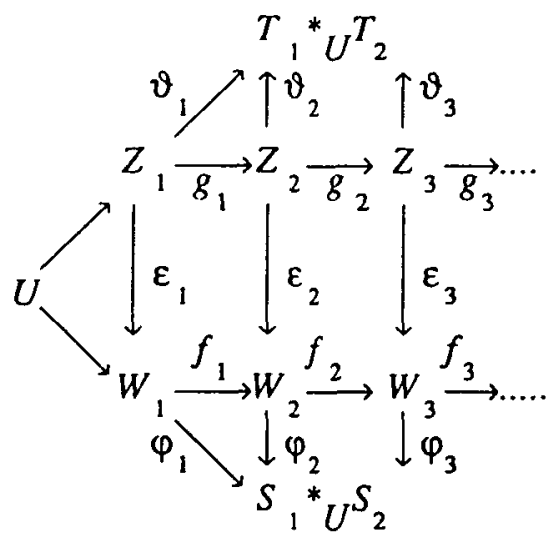


where $\varepsilon_{1}: Z_{1} \rightarrow W_{1}$ is the inclusion, $\varepsilon_{2}: Z_{2} \rightarrow W_{2}$ is given by $\varepsilon_{2}\left(t_{1} \otimes t_{2}\right)=t_{1} \otimes t_{2}$, and in general $\varepsilon_{n}: Z_{n} \rightarrow W_{n}$ is the unique $T_{i}$-map $(i \equiv n(\bmod 2))$ which makes the diagram

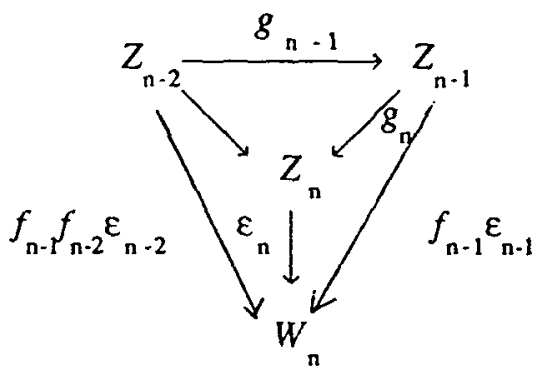

commute.

If we denote the canonical map $T_{1}{ }_{U} T_{2} \rightarrow S_{1} * U S_{2}$ by $\psi$, it is easy to show (see Renshaw [8]) that if each $\varepsilon_{i}$ is one to one then so is $\psi$. Our aim therefore is to show that under certain conditions imposed on $U, S_{i}$ and $T_{i}$, each $\varepsilon_{i}$ is a $U$-monomorphism.

Theorem 2.1. Let $\left[U ; T_{1}, T_{2}\right]$ be an amalgam of submonoids of an amalgam $\left[U ; S_{1}, S_{2}\right]$ such that the inclusions $U \rightarrow T_{i}$ and $T_{i} \rightarrow S_{i}$ are perfect right $U$-monomorphisms, $i=1,2$. Then the canonical map $T_{1} *_{U} T_{2} \rightarrow S_{1}{ }_{U} S_{2}$ is also a perfect $U$-monomorphism.

Proof. Consider the commutative diagram

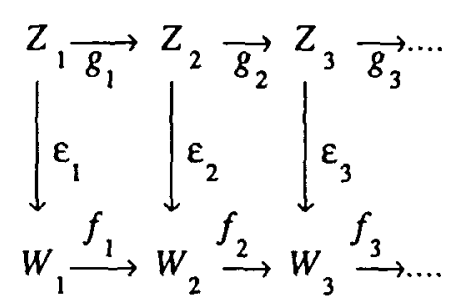

We see that we need only establish the conditions of Theorem 1.9 for the first square and it will then follow by induction that each $\varepsilon_{i}$ is perfect. It is easy to establish that $f_{1}$ and $g_{1}$ are perfect monomorphisms. For example, since $S_{1}$ is flat then $f_{1}$ is one to one; since $S_{1}$ and $S_{2}$ are both flat then $W_{2}=S_{1} \otimes S_{2}$ is flat and since the inclusion $U \rightarrow S_{2}$ is right pure and $S_{1}$ is right flat then $f_{1}$ is right pure. Now $\varepsilon_{1}$ is perfect by assumption. It is easy to check that the tensor product of two perfect monomorphisms is another perfect monomorphism and so $\varepsilon_{2}$ is perfect. Finally, if $X \in U$-ENS, then the commutative diagram

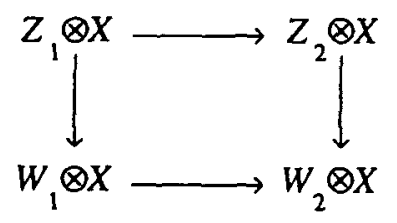


is a pullback, since it can be "redrawn" as

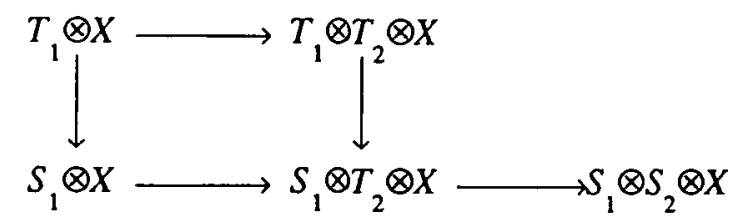

The square is a pullback, by Lemma 1.7 and since $T_{2} \rightarrow S_{2}$ is right pure and $S_{1}$ is right flat then the map $S_{1} \otimes T_{2} \otimes X \rightarrow S_{1} \otimes S_{2} \otimes X$ is one to one. So the conditions of Theorem 1.9 hold and therefore each $\varepsilon_{i}$ is a perfect $U$-monomorphism. It now follows that the map $T_{1}{ }_{U} T_{2} \rightarrow S_{1}{ }_{U} S_{2}$ is pure and since it is known that $S_{1}{ }_{U} S_{2}$ is also flat (Renshaw [10, V.1.4] and Bulman-Fleming and McDowell [1, Theorem 4]) the result then follows. (Note that direct limits of flat $U$-sets are flat.)

Using the well-known fact that amalgamated free products are associative, it is then an easy matter to extend this result to amalgams with a finite index set and then to an arbitrary index set using Renshaw [8, Theorem 1.2].

The next theorem can be proved in a similar way using Theorem 1.10 in place of Theorem 1.9. The details have been omitted.

Theorem 2.2. Let $\left[U ; T_{1}, T_{2}\right]$ be an amalgam of submonoids of an amalgam $\left[U ; S_{1}, S_{2}\right]$ such that $T_{i}, T_{i} / U, S_{i}, S_{i} / U$ and $S_{i} / T_{i}$ are all flat for $i=1,2$. Then the canonical map $T_{1} * T_{U} \rightarrow S_{1}{ }_{U} S_{2}$ is a $U$-monomorphism.

Say that a monoid $U$ is a perfect amalgamation base if

(1) $U$ is an amalgamation base, and

(2) whenever $\left[U ; T_{i}\right]$ is an amalgam of submonoids of $\left[U ; S_{i}\right]$ then $\Pi_{U}^{*} T_{i} \rightarrow \Pi_{U}^{*} S_{i}$ is one to one.

It is now clear that if $U$ is absolutely flat (in other words, every $U$-set is flat) then $U$ is a perfect amalgamation base. Since it is known that inverse semigroups are absolutely flat we can deduce:

Corollary 2.3. Inverse semigroups are perfect amalgamation bases.

It is easy to show that $U$ is absolutely flat if and only if $U$ is absolutely perfect (i.e. the inclusion $U \rightarrow S$ is perfect as a right and left $U$-map, for every containing monoid, $S$ ) (see also, for example, Renshaw [9 or 10]). Hence absolutely perfect monoids are perfect amalgamation bases. The converse of this result was established in Renshaw [8]. Note, however, that we need the inclusions to be perfect both as left $U$-maps and right $U$ maps. For example the two element right zero semigroup, $U$, is such that $U \rightarrow S$ is always perfect as a left $U$-map but not always as a right $U$-map (see Hall [2]). Since perfect amalgamation bases need have both these properties, we deduce that the two element right zero semigroup is not a perfect amalgamation base. Other examples of 
amalgamation bases that are not perfect amalgamation bases can be found among the cyclic semigroups. Hall [2] has shown that every finite cyclic semigroup is an amalgamation base and that the only cyclic semigroups that are absolutely perfect are the groups. Hence the only cyclic, perfect amalgamation bases are the cyclic groups.

Since inverse semigroups are perfect amalgamation bases, it is natural to ask: if $\left[U ; T_{i}\right]$ is an amalgam of subsemigroups of the amalgam $\left[U ; S_{i}\right]$ and if $U, T_{i}$ and $S_{i}$ are all inverse, is it true that $\mathscr{T}^{*}{ }_{U} T_{i}$ the free inverse product of the amalgam $\left[U ; T_{i}\right]$, is embeddable in $\mathscr{T}^{*}{ }_{U} S_{i}$ the free inverse product of the amalgam $\left[U ; S_{i}\right]$ ? The answer is in the affirmative. To see this we note that in any class of semigroups with amalgamated free products, it is straightforward to check the following,

(i) $\left(A *_{U} B\right) *_{S} C \cong A *_{U}\left(B *_{S} C\right)$,

(ii) $U *_{U} D \cong D$,

whenever $U, S, A, B, C$ and $D$ are semigroups such that $U \subseteq A, B, D$ and $S \subseteq B, C$. Now suppose that $\mathscr{C}$ is a class of semigroups with amalgamated free products (e.g. inverse semigroups) and suppose that $\left[U ; T_{1}, T_{2}\right]$ is an amalgam of subsemigroups of $\left[U ; S_{1}, S_{2}\right]$ where all the semigroups belong to $\mathscr{C}$. Then using the above isomorphisms we can deduce that $S_{1} *_{U} S_{2} \cong\left(\left(T_{1} *_{U} T_{2}\right) *{ }_{T_{2}} S_{2}\right){ }^{*} T_{1} S_{1}$ and so if, in $\mathscr{C}, U, T_{1}$ and $T_{2}$ are all amalgamation bases (e.g. if they are all inverse) it follows that $T_{1}{ }^{*}{ }_{U} T_{2}$ can be embedded in $S_{1}{ }_{U} S_{2}$.

\section{Right generalised inverse semigroups}

Lemma 3.1 (Bulman-Fleming and McDowell [1, Lemma 1.2]). $x \otimes y=x^{\prime} \otimes y^{\prime}$ in $X \otimes Y$ if and only if there exists $x_{1}, \ldots, x_{n}$ in $X, y_{2}, \ldots, y_{n}$ in $Y, u_{1}, \ldots, u_{n}$ and $v_{1}, \ldots, v_{n}$ in $U$ such that

$$
\begin{array}{rlrl}
x & =x_{1} u_{1} & u_{1} y=v_{1} y_{2} \\
x_{1} v_{1} & =x_{2} u_{2} & u_{2} y_{2}=v_{2} y_{3} \\
\cdots \cdots & \\
x_{n-1} v_{n-1} & =x_{n} u_{n} & u_{n} y_{n}=y^{\prime} \\
x_{n} v_{n} & =x^{\prime} & &
\end{array}
$$

A set of equations such as those above is called a $U$-scheme over $X$ and $Y$ joining $(x, y)$ to $\left(x^{\prime}, y^{\prime}\right)$.

In what follows we will denote by ${ }^{1} U$ the monoid obtained from the semigroup $U$ by adjoining an identity, 1, regardless of whether $U$ already has an identity. See Renshaw [9] for results concerning amalgams of this type of monoid. A semigroup, $S$, is called right generalised inverse if it is regular and $e f g=f e g$ for all idempotents $e, f$ and $g$. Hall [3, Theorem 4] proved that if $U$ is a regular subsemigroup of a semigroup $S$ such that $e f s=f e s$ for all idempotents $e, f \in S$ and for all $s \in S$, then ${ }^{1} U \rightarrow{ }^{1} S$ is perfect as a left ${ }^{1} U$-map. In particular, this will be true if $U$ is an inverse semigroup or if both $U$ and $S$ are right generalised inverse semigroups. We extend this result as follows: 
Theorem 3.2. Let $U$ be a regular subsemigroup of a semigroup $T$ and $T$ a subsemigroup of a semigroup $S$ and suppose that efs $=$ fes for all $s \in S$, and all $e, f \in E(T)$. Then the inclusion ${ }^{1} T \rightarrow{ }^{1} S$ is a left pure ${ }^{1} U$-monomorphism.

Proof. Let $Z \in$ ENS- $^{-1} U$ and suppose that $z \otimes t=z^{\prime} \otimes t^{\prime}$ in $Z \otimes_{1_{U}}{ }^{1} S$. Then we can construct a ${ }^{1} U$-scheme of the form

$$
\begin{array}{rlr}
z & =z_{1} u_{1} & u_{1} t=v_{1} s_{2}, \\
z_{1} v_{1} & =z_{2} u_{2} & u_{2} s_{2}=v_{2} s_{3}, \\
\ldots \ldots & \\
z_{n-1} v_{n-1} & =z_{n} u_{n} & u_{n} s_{n}=v_{n} t^{\prime} \\
z_{n} v_{n} & =z^{\prime} &
\end{array}
$$

where $z_{i} \in Z, u_{i}, v_{i} \in{ }^{1} U, s_{i} \in{ }^{1} S$ and $t, t^{\prime} \in{ }^{1} T$. We can assume, without loss of generality, that each $u_{i}, v_{i} \in U$ (otherwise a smaller ${ }^{1} U$-scheme could be formed). We therefore need to consider three cases:

Case $I, \quad t, t^{\prime} \in T$ and each $s_{i} \in S$.

Let us define

$$
\begin{array}{ll}
x_{0}=1, x_{1}=v_{1}^{-1}, x_{i}=v_{i}^{-1} u_{l} x_{i-1} & 2 \leqq i \leqq n \\
y_{0}=1, y_{1}=u_{n}^{-1}, y_{i}=u_{n-i+1}^{-1} v_{n-i+1} y_{i-1} & 2 \leqq i \leqq n,
\end{array}
$$

where $u_{i}^{-1}$ and $v_{i}^{-1}$ are fixed inverses (in $U$ ) of $u_{i}$ and $v_{i}$. So in other words,

$$
\begin{gathered}
x_{i}=v_{i}^{-1} u_{i} v_{i-1}^{-1} u_{i-1} \ldots v_{2}^{-1} u_{2} v_{1}^{-1} \\
y_{i}=u_{n-i+1}^{-1} v_{n-i+1} u_{n-i+2}^{-1} \ldots u_{n-1}^{-1} v_{n-1} u_{n}^{-1}
\end{gathered}
$$

Define

$$
\begin{gathered}
x_{0}^{-1}=1, \quad x_{1}^{-1}=v_{1}, \quad x_{i}^{-1}=v_{1} u_{2}^{-1} \ldots u_{i}^{-1} v_{i} \\
y_{0}^{-1}=1, \quad y_{1}^{-1}=u_{n}, \quad y_{i}^{-1}=u_{n} v_{n-1}^{-1} \ldots v_{n-i+1}^{-1} u_{n-i+1}
\end{gathered}
$$

We shall prove:

\section{Lemma.}

(i) $u_{1} t=v_{1} x_{1} u_{1} t$

(ii) $u_{i} x_{i-1} u_{1} t=u_{i} x_{i-1} x_{i}^{-1} s_{i+1}, \quad 2 \leqq i \leqq n \quad$ (where $s_{n+1}=t^{\prime}$ ),

(iii) $u_{i} x_{i-1} u_{1} t=v_{i} x_{i} u_{1} t, \quad 2 \leqq i \leqq n$, 
(iv) $v_{n} t^{\prime}=u_{n} y_{1} v_{n} t^{\prime}$

(v) $v_{i} y_{n-i} v_{n} t^{\prime}=v_{i} y_{n-i} y_{n-i+1}^{-1} s_{i}, \quad 1 \leqq i \leqq n-1 \quad$ (where $s_{1}=t$ ),

(vi) $v_{i} y_{n-i} v_{n} t^{\prime}=u_{i} y_{n-i+1} v_{n} t^{\prime}, \quad 1 \leqq i \leqq n-1$,

(vii) $v_{n} x_{n} u_{1} t=u_{n} x_{n-1} x_{n}^{-1} t^{\prime}$,

(viii) $v_{i} x_{i} x_{n}^{-1} t^{\prime}=u_{i} x_{i-1} x_{n}^{-1} t^{\prime}, \quad 2 \leqq i \leqq n-1$,

(ix) $v_{1} x_{1} x_{n}^{-1} t^{\prime}=v_{1} y_{n-1} v_{n} t^{\prime}$.

Proof of lemma.

(i) $u_{1} t=v_{1} s_{2}=v_{1} v_{1}^{-1} v_{1} s_{2}=v_{1} v_{1}^{-1} u_{1} t=v_{1} x_{1} u_{1} t$.

(ii) We use induction. When $i=2$, we have

$$
\begin{aligned}
u_{2} x_{1} u_{1} t & =u_{2} v_{1}^{-1} u_{1} t=u_{2} v_{1}^{-1} v_{1} s_{2}=u_{2} u_{2}^{-1} u_{2} v_{1}^{-1} v_{1} s_{2} \\
& =u_{2} v_{1}^{-1} v_{1} u_{2}^{-1} u_{2} s_{2}=u_{2} x_{1} v_{1} u_{2}^{-1} v_{2} s_{3}=u_{2} x_{1} x_{2}^{-1} s_{3}
\end{aligned}
$$

Now assume the result is true for $i=k$, say and consider

$$
\begin{aligned}
u_{k+1} x_{k} u_{1} t & =u_{k+1} v_{k}^{-1} u_{k} x_{k-1} u_{1} t=u_{k+1} v_{k}^{-1} u_{k} x_{k-1} x_{k}^{-1} s_{k+1}, \text { by induction } \\
& =u_{k+1} u_{k+1}^{-1} u_{k+1} v_{k}^{-1} u_{k} x_{k-1} x_{k}^{-1} s_{k+1}=u_{k+1} u_{k+1}^{-1} u_{k+1} x_{k} x_{k}^{-1} s_{k+1} \\
& =u_{k+1} x_{k} x_{k}^{-1} u_{k+1}^{-1} u_{k+1} s_{k+1}=u_{k+1} x_{k} x_{k}^{-1} u_{k+1}^{-1} v_{k+1} s_{k+2} \\
& =u_{k+1} x_{k} x_{k+1}^{-1} s_{k+2}
\end{aligned}
$$

The result follows by induction.

(iii)

$$
\begin{aligned}
u_{i} x_{i-1} u_{1} t & =u_{i} x_{i-1} x_{i}^{-1} s_{i+1}=u_{i} x_{i-1} x_{i-1}^{-1} u_{i}^{-1} v_{i} s_{i+1} \\
& =u_{i} x_{i-1} x_{i-1}^{-1} u_{i}^{-1} v_{i} v_{i}^{-1} v_{i} s_{i+1}=v_{i} v_{i}^{-1} u_{i} x_{i-1} x_{i-1}^{-1} u_{i}^{-1} v_{i} s_{i+1} \\
& =v_{i} v_{i}^{-1} u_{i} x_{i-1} u_{1} t=v_{i} x_{i} u_{1} t
\end{aligned}
$$

(iv)

$$
v_{n} t^{\prime}=u_{n} s_{n}=u_{n} u_{n}^{-1} u_{n} s_{n}=u_{n} u_{n}^{-1} v_{n} t^{\prime}=u_{n} y_{1} v_{n} t^{\prime}
$$

(v) When $i=n-1$, we have

$$
\begin{aligned}
v_{n-1} y_{1} v_{n} t^{\prime} & =v_{n-1} u_{n}^{-1} v_{n} t^{\prime}=v_{n-1} u_{n}^{-1} u_{n} s_{n}=v_{n-1} v_{n-1}^{-1} v_{n-1} u_{n}^{-1} u_{n} s_{n} \\
& =v_{n-1} u_{n}^{-1} u_{n} v_{n-1}^{-1} v_{n-1} s_{n}=v_{n-1} y_{1} u_{n} v_{n-1}^{-1} u_{n-1} s_{n-1} \\
& =v_{n-1} y_{1} y_{2}^{-1} s_{n-1} .
\end{aligned}
$$


Assume the result is true for $i=k$ and consider

$$
\begin{aligned}
v_{k-1} y_{n-k+1} v_{n} t^{\prime} & =v_{k-1} u_{k}^{-1} v_{k} y_{n-k} v_{n} t^{\prime} \\
& =v_{k-1} u_{k}^{-1} v_{k} y_{n-k} y_{n-k+1}^{-1} s_{k}, \quad \text { by induction } \\
& =v_{k-1} v_{k-1}^{-1} v_{k-1} y_{n-k+1} y_{n-k+1}^{-1} s_{k}, \\
& =v_{k-1} y_{n-k+1} y_{n-k+1}^{-1} v_{k-1}^{-1} v_{k-1} s_{k}, \\
& =v_{k-1} y_{n-k+1} y_{n-k+1}^{-1} v_{k-1}^{-1} u_{k-1} s_{k-1}, \\
& =v_{k-1} y_{n-k+1} y_{n-k+2}^{-1} s_{k-1}
\end{aligned}
$$

The result follows by induction.

$$
\begin{aligned}
v_{i} y_{n-i} v_{n} t^{\prime} & =v_{i} y_{n-i} y_{n-i+1}^{-1} s_{i}=v_{l} y_{n-i} y_{n-i}^{-1} v_{i}^{-1} u_{i} s_{i} \\
& =v_{i} y_{n-i} y_{n-i}^{-1} v_{i}^{-1} u_{i} u_{i}^{-1} u_{i} s_{i}=u_{i} u_{i}^{-1} v_{i} y_{n-i} y_{n-i}^{-1} v_{i}^{-1} u_{i} s_{i} \\
& =u_{i} u_{i}^{-1} v_{i} y_{n-i} v_{n} t^{\prime}=u_{i} y_{n-i+1} v_{n} t^{\prime}
\end{aligned}
$$

$$
v_{n} x_{n} u_{1} t=u_{n} x_{n-1} x_{n}^{-1} t^{\prime} \quad \text { by (ii) and (iii). }
$$

(viii) First, notice that $x_{n}^{-1}=x_{i}^{-1} y_{n-i} v_{n}, 1 \leqq i \leqq n-1$.

$$
\begin{aligned}
v_{i} x_{i} x_{n}^{-1} t^{\prime} & =v_{i} v_{i}^{-1} u_{i} x_{i-1} x_{i}^{-1} y_{n-i} v_{n} t^{\prime}=v_{i} v_{i}^{-1} u_{i} x_{i-1} x_{i-1}^{-1} u_{i}^{-1} v_{i} y_{n-i} v_{n} t^{\prime} \\
& =u_{i} x_{i-1} x_{i-1}^{-1} u_{i}^{-1} v_{i} v_{i}^{-1} v_{i} y_{n-i} v_{n} t^{\prime}=u_{i} x_{i-1} x_{i-1}^{-1} u_{i}^{-1} v_{i} y_{n-i} v_{n} t^{\prime} \\
& =u_{i} x_{i-1} x_{i}^{-1} y_{n-i} v_{n} t^{\prime}=u_{i} x_{i-1} x_{n}^{-1} t^{\prime}
\end{aligned}
$$

(ix)

$$
\begin{aligned}
v_{1} x_{1} x_{n}^{-1} t^{\prime}= & v_{1} v_{1}^{-1} x_{1}^{-1} y_{n-1} v_{n} t^{\prime}, \text { since } x_{n}^{-1}=x_{1}^{-1} y_{n-1} v_{n} \\
& =v_{1} v_{1}^{-1} v_{1} y_{n-1} v_{n} t^{\prime}=v_{1} y_{n-1} v_{n} t^{\prime}
\end{aligned}
$$

If we now return to the proof of the theorem and put $a_{i}=x_{i} u_{1} t, b_{i}=x_{i} x_{n}^{-1} t^{\prime}$ and $c_{i}=y_{n-i+1} v_{n} t^{\prime}$, then it is easy to check that the following is a ${ }^{1} U$-scheme over $Z$ and ${ }^{1} T$ joining $(z, t)$ and $\left(z^{\prime}, t^{\prime}\right)$ and so $z \otimes t=z^{\prime} \otimes t^{\prime}$ in $Z \otimes_{1}{ }^{1} T$ as required.

$$
\begin{array}{rlrl}
z & =z_{1} u_{1} \\
z_{1} v_{1} & =z_{2} u_{2} & u_{1} t=v_{1} a_{1} \\
u_{2} a_{1} & =v_{2} a_{2}
\end{array}
$$




$$
\begin{aligned}
& z_{i} v_{i}=z_{i+1} u_{i+1} \quad u_{i+1} a_{i}=v_{i+1} a_{i+1} \\
& z_{n-2} v_{n-2}=z_{n-1} u_{n-1} \quad u_{n-1} a_{n-2}=v_{n-1} a_{n-1} \\
& z_{n-1} v_{n-1}=z_{n} u_{n} \quad u_{n} a_{n-1}=u_{n} b_{n-1} \\
& z_{n} u_{n}=z_{n-1} v_{n-1} \quad v_{n-1} b_{n-1}=u_{n-1} b_{n-2} \\
& z_{i} u_{i}=z_{i-1} v_{i-1} \quad v_{i-1} b_{i-1}=u_{i-1} b_{i-2} \\
& z_{3} u_{3}=z_{2} v_{2} \quad v_{2} b_{2}=u_{2} b_{1} \\
& z_{2} u_{2}=z_{1} v_{1} \quad v_{1} b_{1}=v_{1} c_{2} \\
& z_{1} v_{1}=z_{2} u_{2} \quad u_{2} c_{2}=v_{2} c_{3} \\
& z_{i} v_{i}=z_{i+1} u_{i+1} \quad u_{i+1} c_{i+1}=v_{i+1} c_{i+2} \\
& z_{n-1} v_{n-1}=z_{n} u_{n} \quad u_{n} c_{n}=v_{n} t^{\prime} \\
& z_{n} v_{n}=z^{\prime}
\end{aligned}
$$

Case II Some of the $s_{i}=1$.

Suppose that $i$ is the smallest index with $s_{i}=1$ and that $j$ is the largest index with $s_{j}=1$. Put $x=v_{i-1} x_{i-1} x_{i-1}^{-1}$ and $y=u_{j} y_{n-j+1} y_{n-j+1}^{-1}$ and notice that $x, y \in U$. Then it is straightforward to check that the following is a ${ }^{1} U$-scheme over $Z$ and ${ }^{1} T$ :

$$
\begin{aligned}
& z=z_{1} u_{1} \quad u_{1} t=v_{1} a_{1} \\
& z_{1} v_{1}=z_{2} u_{2} \quad u_{2} a_{1}=v_{2} a_{2} \\
& z_{i-2} v_{i-2}=z_{i-1} u_{i-1} \quad u_{i-1} a_{i-2}=x \cdot 1 \\
& z_{i-1} x=\left(z_{i-1} x\right)
\end{aligned}
$$

and so we have $z \otimes t=z_{i-1} x \otimes 1$ in $Z \otimes{ }^{1} T$. In a similar way it is again easy to check the following is a ${ }^{1} U$-scheme over $Z$ and ${ }^{1} T$ :

$$
\begin{array}{rlrl}
z^{\prime} & =z_{n} v_{n} & v_{n} t^{\prime} & =u_{n} c_{n} \\
z_{n} u_{n} & =z_{n-1} v_{n-1} & & v_{n-1} c_{n}=u_{n-1} c_{n-1} \\
z_{j+1} u_{j+1} & =z_{j} v_{j} & & \\
z_{j} y & =\left(z_{j} y\right) & v_{j} c_{j+1}=y \cdot 1
\end{array}
$$


and so $z^{\prime} \otimes t^{\prime}=z_{j} y \otimes 1$ in $Z \otimes{ }^{1} T$. But $z \otimes t=z^{\prime} \otimes t^{\prime}$ in $Z \otimes{ }^{1} S$ and so $z_{i-1} x \otimes 1=z_{j} y \otimes 1$ in $Z \otimes{ }^{1} S$. By Hall [3, Theorem 2] we deduce that $z_{i-1} x=z_{j} y$ and so, in $Z \otimes{ }^{1} T$ we have $z \otimes t=z_{i-1} x \otimes 1=z_{j} y \otimes 1=z^{\prime} \otimes t^{\prime}$ in $Z \otimes{ }^{1} T$, as required.

Case III $t-1$ or $t^{\prime}=1$.

Suppose, without loss of generality that $t^{\prime}=1$. If $t=1$, then by Hall [3, Theorem 2] it follows that $z=z^{\prime}$ and so $z \otimes 1=z^{\prime} \otimes 1$ in $Z \otimes{ }^{1} T$. If $t \neq 1$, and $s_{i}=1$ for some $i$, then by the above argument, there exists $z^{\prime \prime} \in Z$ with $z \otimes t=z^{\prime \prime} \otimes 1$ in $Z \otimes{ }^{1} T$. Hence $z^{\prime} \otimes 1=$ $z^{\prime \prime} \otimes 1$ in $Z \otimes{ }^{1} S$ and so by Hall [3, Theorem 2] again, $z^{\prime}=z^{\prime \prime}$ giving $z \otimes t=z^{\prime} \otimes 1$ in $Z \otimes{ }^{1} T$ as required. Suppose then that $t \neq 1$ and that each $s_{i} \neq 1$. In this case we see that the original scheme can be rewritten as:

$$
\begin{aligned}
& z=z_{1} u_{1} \quad u_{1} t=v_{1} s_{2}, \\
& z_{1} v_{1}=z_{2} u_{2} \quad u_{2} s_{2}=v_{2} s_{3} \text {, } \\
& z_{n-1} v_{n-1}=z_{n} u_{n} \quad u_{n} s_{n}=v_{n}\left(v_{n}^{-1} v_{n}\right) \\
& z_{n} v_{n}=z^{\prime}
\end{aligned}
$$

and so using the procedure in Case I, we can deduce that $z \otimes t=z^{\prime} \otimes v_{n}^{-1} v_{n}$ in $Z \otimes{ }^{1} T$. But $z^{\prime} \otimes v_{n}^{-1} v_{n}=z^{\prime} v_{n}^{-1} v_{n} \otimes 1=z_{n} v_{n} v_{n}^{-1} v_{n} \otimes 1=z_{n} v_{n} \otimes 1=z^{\prime} \otimes 1$ in $Z \otimes{ }^{1} T$. The proof of Theorem 3.2 is complete.

Using Hall [3, Theorem 4] and the dual of Theorem 2.1, we can now deduce immediately:

Theorem 3.3. Let $U$ be a regular semigroup and let $\left[U ; T_{i}\right]$ be an amalgam of subsemigroups of an amalgam $\left[U ; S_{i}\right]$ such that $e_{i} f_{i} s_{i}=f_{i} e_{i} s_{i}$ for all $i$ and for all $s_{i} \in S_{i}$ and all idempotents $e_{i}, f_{i} \in T_{i}$. Then $\Pi_{v}^{*} T_{i} \rightarrow \Pi_{v}^{*} S_{i}$ is one to one. In particular, the conclusion holds if $U$ is inverse or if $U, T_{i}$ and $S_{i}$ are all right generalised inverse.

Acknowledgement. The author would like to thank the referee for pointing out an error in an earlier version of this paper.

\section{REFERENCES}

3. S. Bulman-Fleming and K. McDowell, Absolutely flat semigroups, Pacific J. Math. 107 (1983), 319-333.

2. T. E. Hall, Representation extension and amalgamation for semigroups, Quart. J. Math. Oxford (2) 29 (1978), 309-334.

3. T. E. Hall, Generalised inverse semigroups and amalgamation, in Semigroups (T. E. Hall, P. R. Jones and G. B. Preston, eds., Academic Press, New York, 1980), 145-157. 
4. J. M. HowIE, An Introduction to Semigroup Theory (London Mathematical Society Monographs 7, Academic Press, London, 1976).

5. J. M. HowIE, Epimorphisms and amalgamations: A survey of recent progress, Coll. Math. Soc. János Bolyai 39 (1981), 63-82.

6. J. M. HowIE, Subsemigroups of amalgamated free products of semigroups, Proc. London Math. Soc. (3) 13 (1963), 672-686.

7. J. Renshaw, Flatness and amalgamation in monoids, J. London Math. Soc. (2) 33 (1986), 73-88.

8. J. Renshaw, Perfect amalgamation bases, J. Algebra, to appear.

9. J. Renshaw, Extension and amalgamation in monoids and semigroups, Proc. London Math. Soc. (3) 52 (1986), 119-141.

10. J. Renshaw, Thesis (University of St. Andrews, 1985).

Faculty of Mathematical Studies

UNIVERSITY OF SOUTHAMPTON

SOUTHAMPTON

SO9 $5 \mathrm{NH}$ 